\title{
New upper and lower bounds, the iteration algorithm for the solution of the discrete algebraic Riccati equation
}

Juan Zhang and Jianzhou Liu*

"Correspondence: liujz@xtu.edu.cn Department of Mathematics and Computational Science, Xiangtan University, Xiangtan, Hunan 411105 , P.R. China

\begin{abstract}
In this paper, applying the properties of matrix Schur complement and matrix inverse, via some matrix equalities and inequalities, we present new lower and upper solution bounds of the discrete algebraic Riccati equation. Then, by the compressed image principle and a matrix norm inequality, we offer an existence uniqueness condition and a fixed point iteration algorithm for the solution of the discrete algebraic Riccati equation. Finally, a corresponding numerical example demonstrates the effectiveness of the developed results.
\end{abstract}

Keywords: matrix bound; discrete algebraic Riccati equation; Schur complement; eigenvalue

\section{Introduction and preliminaries}

The discrete algebraic Riccati equation has many applications in the process of obtaining optimal control and determining system stability $[1,2]$. For example, consider the following linear discrete system (see [2]):

$$
x(t+1)=A x(t)+B u(t)
$$

where $x(t) \in \mathbb{R}^{n}$ is the state variable, $u(t) \in \mathbb{R}^{m}$ is the input variable, $A \in \mathbb{R}^{n \times n}$ is the system matrix, $B \in \mathbb{R}^{n \times m}$ is the input matrix.

The system (1.1) is associated with the linear quadratic optimal control problem of minimizing the quadratic performance index

$$
\widetilde{J}=\sum_{t=0}^{\infty}\left(\begin{array}{l}
x(t) \\
u(t)
\end{array}\right)^{T}\left(\begin{array}{ll}
Q & S^{T} \\
S & G
\end{array}\right)\left(\begin{array}{l}
x(t) \\
u(t)
\end{array}\right),
$$

where $S \in \mathbb{R}^{m \times n}, G \in \mathbb{R}^{m \times m}, Q \in \mathbb{R}^{n \times n}$ are symmetric positive definite matrices, then the problem switches to how to solve the symmetric positive definite solution $P \in \mathbb{R}^{n \times n}$ of the following equation:

$$
P=A^{T} P A+Q-\left(A^{T} P B+S^{T}\right)\left(G+B^{T} P B\right)^{-1}\left(B^{T} P A+S\right) .
$$

\section{Springer}


If $S=0, G=I_{m},(1.2)$ becomes the discrete algebraic Riccati matrix equation

$$
P=A^{T} P A-A^{T} P B\left(I+B^{T} P B\right)^{-1} B^{T} P A+Q .
$$

In many practical control problems, to study the discrete algebraic Riccati equation (1.3), we usually assume that $Q$ is positive semi-definite. When considering certain $H$-infinity problems and LQG problems and so on in some discrete control linear systems, the assumption that $Q$ is positive definite is only required. Certainly, it is difficult to discuss this equation on the assumption that $Q$ is positive semi-definite. Thus, there are many scholars to research this equation on the assumption that $Q$ is positive definite [3-6].

As the discrete algebraic Riccati equations (1.2) and (1.3) play an important role in many control analysis and design problems (Bernstein, 2005 [1]; Kojima et al., 2006 [2]), discussing these equations becomes a heated topic. For one thing, it is important to solve these equations (Kojima et al., 2006 [2]). It is difficult to obtain their solutions, and there are few iterative algorithms for getting the solutions of these equations (Komaroff, 1994 [3]). Reference [3] derives the iterative solution algorithm on the assumption that for the discrete algebraic Riccati equations (1.3) there exists a positive definite solution. In certain control problems, if the original system is assumed to be stabilizable, the exact solutions of the discrete algebraic Riccati equations (1.2) and (1.3) are not often required, while a reasonably tight solution bound will only be required such as the stabilization of jump linear systems (Fang and Loparo, 2002 [7]) and the quadratic optimization problem for a class of singularly perturbed stochastic systems (Dragan, 2005 [8]). Hence, in recent years, many researchers paid much attention to studying the discrete algebraic Riccati equations (1.2) and (1.3). Those include a recursive solution (Assimakis et al., 2003 [9]), a matrix inequality (Saberi, 1998 [10]), prediction, estimation, and smoothing error covariance matrices (Assimakis and Adam, 2013 [11]), iterative and algebraic algorithms (Assimakis and Adam, 2014 [12]; Chiang et al., 2010 [13]), matrix bounds (Choi, 2001 [14]; Kim and Park, 1999 [15]; Gao et al., 2002 [16]; Komaroff, 1994, 1992 [3, 17]; Lee and Chang, 1998 [18]; Lee, 1996, 1997, 2003 [19-22], Liu and Zhang, 2011, 2012 [23-25], Zhang and Liu, 2012 [26, 27]), eigenvalue bounds (Garloff, 1986 [5]; Lee, 2004 [6]), trace bounds (Kim et al., 1993 [28]), summation bounds (Komaroff and Shahian, 1992 [29]), norm bounds (Patel and Toda, 1978 [30]), and perturbation bounds (Hasanov et al., 2004 [31]; Hasanov, 2010 [32]). The most general bounds are the solution bounds, as they can directly offer the other types of bounds mentioned. Thus, based on the above, there are three problems to be considered as follows:

(1) When does there exist a positive definite solution of the discrete algebraic Riccati equation (1.3)?

(2) If the discrete algebraic Riccati equation (1.3) possesses a positive definite solution under certain conditions, in what range is the solution unique? In addition, how do we estimate the upper and lower solution bounds of this equation?

(3) If the discrete algebraic Riccati equation (1.3) possesses a unique positive definite solution in a certain range, how do we design the iterative solution algorithms? Further, we need to discuss the solution bounds affecting the iterative algorithms.

Therefore, in this paper, we present new lower and upper solution bounds of the discrete algebraic Riccati equation (1.3). Then we offer an existence uniqueness condition and a fixed iterative algorithm for the solution of the discrete algebraic Riccati equation 
(1.3). Finally, a corresponding numerical example demonstrates the effectiveness of the developed results.

Throughout this paper, we use the following symbol conventions. Let $\mathbb{R}^{n \times m}$ and $N^{+}$denote the set of $n \times m$ real matrices and positive integers. For $X=\left(x_{i j}\right) \in \mathbb{R}^{m \times n}$, let $X^{T}$, $\|X\|$ denote the transpose and the spectral norm of $X$, respectively. If $X \in \mathbb{R}^{n \times n}, X^{-1}$ denotes the inverse of $X$. If $X, Y \in \mathbb{R}^{n \times n}$, the inequality $X>(\geq) 0$ means that $X$ is a symmetric positive (semi-) definite matrix and the inequality $X>(\geq) Y$ means $X-Y$ is a symmetric positive (semi-) definite matrix. Suppose $X \in \mathbb{R}^{n \times n}$ is an arbitrary symmetric matrix, we assume that the eigenvalues of $X$ are arranged so that $\lambda_{1}(X) \geq \lambda_{2}(X) \geq \cdots \geq \lambda_{n}(X)$. For $X \in \mathbb{R}^{n \times n}$, suppose the singular values of $X$ are arranged so that $\sigma_{1}(X) \geq \sigma_{2}(X) \geq \cdots \geq \sigma_{n}(X)$. The identity matrix with appropriate dimensions is represented by $I$.

Let $N=\{1,2, \ldots, n\}$. For nonempty index sets $\alpha, \beta \subseteq N$ whose elements are both conventionally arranged in increasing order, we denote by $|\alpha|$ the cardinality of $\alpha$ and by $\alpha^{c}=N-\alpha$ the complement of $\alpha$ in $N$. We write $X(\alpha, \beta)$ to mean the submatrix of $X \in R^{n \times n}$ lying in the rows indexed by $\alpha$ and the columns indexed by $\beta . X(\alpha, \alpha)$ is abbreviated to $X(\alpha)$. Assuming that $X(\alpha)$ is nonsingular, denote the Schur complement with respect to $X(\alpha)$ by

$$
X / \alpha=X / X(\alpha)=X\left(\alpha^{c}\right)-X\left(\alpha^{c}, \alpha\right)[X(\alpha)]^{-1} X\left(\alpha, \alpha^{c}\right) .
$$

The following lemmas are used to prove the main results.

Lemma 1.1 (Zhang [33], p.19, Theorem 1.2) Let $X \in \mathbb{R}^{n \times n}$ be partitioned as

$$
X=\left(\begin{array}{ll}
E & F \\
G & H
\end{array}\right)
$$

and suppose both $X$ and $E \in \mathbb{R}^{m \times m}$ are nonsingular matrices. Then $X / E$ is nonsingular and

$$
X^{-1}=\left(\begin{array}{cc}
E^{-1}+E^{-1} F S^{-1} G E^{-1} & -E^{-1} F S^{-1} \\
-S^{-1} G E^{-1} & S^{-1}
\end{array}\right),
$$

where $S=H-G E^{-1} F=X / E$.

Lemma 1.2 (Zhang [33], p.25, Case 5) If the matrix $X$ is defined as Lemma 1.1, choosing $\alpha=\{1,2, \ldots, m\}$, then

$$
(X / \alpha)^{-1}=X^{-1}\left(\alpha^{c}\right)
$$

Lemma 1.3 (Lee [20]) Let $P$ be the positive semi-definite solution of the discrete algebraic Riccati equation (1.3), and $\sigma_{1}^{2}(A)<1+\sigma_{n}^{2}(B) \delta_{1}$, then $P$ has the upper matrix bound

$$
P \leq \frac{\lambda_{1}(Q)}{1+\delta_{1} \sigma_{n}^{2}(B)-\sigma_{1}^{2}(A)} A^{T} A+Q \equiv \eta
$$

where $\delta_{1} \equiv \lambda_{1}\left[A^{T}\left(I+Q B B^{T}\right)^{-1} Q A+Q\right]$. 
Definition 1.1 (Rudin [34]) The pair $(X, d)$ is called a complete metric space if $X$ is a nonempty set whose elements are denoted by $x, y, \ldots$ and assume that on the Cartesian product $X \times X$ a distance function $d$ is defined satisfying the following conditions:

(D0) $d(x, y) \geq 0$,

(Dl) $d(x, y)=0$ if and only if $x=y$,

(D2) $d(x, y)=d(y, x)$,

(D3) $d(x, y) \leq d(x, z)+d(z, y)$,

(D4) every $d$-Cauchy sequence in $X$ is $d$-convergent, i.e., if $x_{n}$ is a sequence in $X$ such that $\lim _{n, m \rightarrow \infty} d\left(x_{n}, x_{m}\right)=0$, then there is an $x \in X$ with $\lim _{n \rightarrow \infty} d\left(x_{n}, x\right)=0$.

Definition 1.2 (Rudin [34]) The pair $(X,\|\cdot\|)$ is called a normed space if $X$ is a real vector space whose elements are denoted by $x, y, z, \ldots$ and assume that for every $x \in X$ there is associated a nonnegative real number $\|x\|$, called the norm of $x$, in such a way that:

(D0) $\|x+y\| \leq\|x\|+\|y\|$,

(Dl) $\|\alpha x\|=|\alpha| \cdot\|x\|$ where $\alpha$ is a scalar,

(D2) $\|x\|>0$ if $x \neq 0$.

Lemma 1.4 (Berinde [35], Theorem B) Let $(X, d)$ be a complete metric space and $F: X \longrightarrow$ $X$ be a strict contraction, i.e., a map satisfying

$$
d(F x, F y) \leq a d(x, y), \quad \text { for all } x, y \in X,
$$

where $0 \leq a<1$ is constant. Then $F$ has a unique fixed point in $X$.

Every normed space may be regarded as a metric space, in which the distance $d(x, y)$ between $x$ and $y$ is $\|x-y\|$. Hence, we get the following conclusion from Definitions 1.1, 1.2 and Lemma 1.4 .

Lemma 1.5 Let $(X,\|\cdot\|)$ be a real Banach space, and $\Omega \subset X$ be a convex, closed and bounded subset and $F: \Omega \longrightarrow \Omega$ be a contraction map, i.e., a map satisfying

$$
\left\|F\left(P_{1}\right)-F\left(P_{2}\right)\right\| \leq p\left\|P_{1}-P_{2}\right\|, \quad \text { for all } P_{1}, P_{2} \in \Omega,
$$

where $0 \leq p<1$ is constant. Then $F$ has a unique fixed point in $\Omega$.

Lemma 1.6 (Zhao et al. [36], Lemma 2.1) If $X, Y \in \mathbb{R}^{n \times n}$, and $0<X \leq Y$, then

$$
\|X\| \leq\|Y\|
$$

\section{New solution bounds for the discrete algebraic Riccati equation (1.3)}

In this section, we first present a lemma (Lemma 2.1). Then new lower and upper solution bounds are deduced from Lemma 2.1.

Lemma 2.1 Define the map

$$
F(P)=A^{T}\left(P^{-1}+B B^{T}\right)^{-1} A+Q
$$


then

$$
F(P)=Q^{\frac{1}{2}}\left[Q^{-\frac{1}{2}} A^{T}\left(P^{-1}+B B^{T}-A(F(P))^{-1} A^{T}\right)^{-1} A Q^{-\frac{1}{2}}+\frac{1}{4} I\right]^{\frac{1}{2}} Q^{\frac{1}{2}}+\frac{1}{2} Q .
$$

Proof By (2.1), then

$$
Q=F(P)-A^{T}\left(P^{-1}+B B^{T}\right)^{-1} A=\left(\begin{array}{cc}
F(P) & A^{T} \\
A & P^{-1}+B B^{T}
\end{array}\right) / \alpha^{c} .
$$

Applying Lemma 1.2 to (2.3) yields

$$
Q=\left[\left(\begin{array}{cc}
F(P) & A^{T} \\
A & P^{-1}+B B^{T}
\end{array}\right)^{-1}(\alpha)\right]^{-1}
$$

which is equivalent to

$$
Q^{-1}=\left(\begin{array}{cc}
F(P) & A^{T} \\
A & P^{-1}+B B^{T}
\end{array}\right)^{-1}(\alpha) .
$$

Introducing Lemma 1.1 to (2.4) gives

$$
Q^{-1}=F(P)^{-1}+F(P)^{-1} A^{T}\left[P^{-1}+B B^{T}-A(F(P))^{-1} A^{T}\right]^{-1} A F(P)^{-1} .
$$

Multiplying (2.5) on the right and on the left by $F(P)$ one gets

$$
F(P) Q^{-1} F(P)=F(P)+A^{T}\left[P^{-1}+B B^{T}-A(F(P))^{-1} A^{T}\right]^{-1} A .
$$

Define

$$
\widetilde{F}(P)=Q^{-\frac{1}{2}} F(P) Q^{-\frac{1}{2}},
$$

multiplying (2.6) on the right and on the left by $Q^{-\frac{1}{2}}$ leads to

$$
\widetilde{F}(P)^{2}-\widetilde{F}(P)+\frac{1}{4} I=Q^{-\frac{1}{2}} A^{T}\left[P^{-1}+B B^{T}-A(F(P))^{-1} A^{T}\right]^{-1} A Q^{-\frac{1}{2}}+\frac{1}{4} I,
$$

from which one infers that

$$
\left(\widetilde{F}(P)-\frac{1}{2} I\right)^{2}=Q^{-\frac{1}{2}} A^{T}\left[P^{-1}+B B^{T}-A(F(P))^{-1} A^{T}\right]^{-1} A Q^{-\frac{1}{2}}+\frac{1}{4} I .
$$

From (2.1), we get

$$
F(P) \geq Q
$$

i.e.,

$$
(F(P))^{-1} \leq Q^{-1}
$$


In terms of (2.9), it is evident that

$$
F(P) \geq Q>\frac{1}{2} Q
$$

or, equivalently

$$
\widetilde{F}(P) \geq \frac{1}{2} I
$$

Combining (2.8) with (2.11) shows that

$$
\widetilde{F}(P)-\frac{1}{2} I=\left[Q^{-\frac{1}{2}} A^{T}\left[P^{-1}+B B^{T}-A(F(P))^{-1} A^{T}\right]^{-1} A Q^{-\frac{1}{2}}+\frac{1}{4} I\right]^{\frac{1}{2}},
$$

i.e.,

$$
\widetilde{F}(P)=\left[Q^{-\frac{1}{2}} A^{T}\left[P^{-1}+B B^{T}-A(F(P))^{-1} A^{T}\right]^{-1} A Q^{-\frac{1}{2}}+\frac{1}{4} I\right]^{\frac{1}{2}}+\frac{1}{2} I .
$$

Substituting (2.7) into (2.12) gives

$$
F(P)=Q^{\frac{1}{2}}\left[Q^{-\frac{1}{2}} A^{T}\left(P^{-1}+B B^{T}-A(F(P))^{-1} A^{T}\right)^{-1} A Q^{-\frac{1}{2}}+\frac{1}{4} I\right]^{\frac{1}{2}} Q^{\frac{1}{2}}+\frac{1}{2} Q
$$

which completes the proof.

Theorem 2.1 Let $P$ be the positive definite solution of the discrete algebraic Riccati equation (1.3), then P has the lower matrix bound

$$
P \geq Q^{\frac{1}{2}}\left[Q^{-\frac{1}{2}} A^{T}\left(Q^{-1}+B B^{T}\right)^{-1} A Q^{-\frac{1}{2}}+\frac{1}{4} I\right]^{\frac{1}{2}} Q^{\frac{1}{2}}+\frac{1}{2} Q \equiv P_{1}
$$

Proof From (1.3), we have

$$
P=A^{T} P\left[P^{-1}-B\left(I+B^{T} P B\right)^{-1} B^{T}\right] P A+Q
$$

Let

$$
X=\left(\begin{array}{cc}
P^{-1} & B \\
B^{T} & I+B^{T} P B
\end{array}\right)
$$

according to Lemmas 1.1 and 1.2, (2.14) changes to

$$
\begin{aligned}
& P=A^{T} P\left[\left(\begin{array}{cc}
P^{-1} & B \\
B^{T} & I+B^{T} P B
\end{array}\right) / \alpha^{c}\right] P A+Q \\
& =A^{T} P\left[\left(\begin{array}{cc}
P^{-1} & B \\
B^{T} & I+B^{T} P B
\end{array}\right)^{-1}(\alpha)\right]^{-1} P A+Q \\
& =A^{T} P\left[\left(\begin{array}{cc}
P+P B(X / \alpha)^{-1} B^{T} P & -P B(X / \alpha)^{-1} \\
-(X / \alpha)^{-1} B^{T} P & (X / \alpha)^{-1}
\end{array}\right)(\alpha)\right]^{-1} P A+Q \text {. }
\end{aligned}
$$


Since

$$
X / \alpha=I+B^{T} P B-B^{T}\left(P^{-1}\right)^{-1} B=I,
$$

(2.15) becomes

$$
P=A^{T} P\left(P+P B B^{T} P\right)^{-1} P A+Q=A^{T}\left(P^{-1}+B B^{T}\right)^{-1} A+Q .
$$

By (2.16), choosing $F(P)=P$ in Lemma 2.1, we have

$$
P=Q^{\frac{1}{2}}\left[Q^{-\frac{1}{2}} A^{T}\left(P^{-1}+B B^{T}-A P^{-1} A^{T}\right)^{-1} A Q^{-\frac{1}{2}}+\frac{1}{4} I\right]^{\frac{1}{2}} Q^{\frac{1}{2}}+\frac{1}{2} Q .
$$

From (2.16), we get

$$
P \geq Q
$$

i.e.,

$$
P^{-1} \leq Q^{-1}
$$

In the light of (2.18), (2.17) turns to

$$
\begin{aligned}
P & \geq Q^{\frac{1}{2}}\left[Q^{-\frac{1}{2}} A^{T}\left(P^{-1}+B B^{T}\right)^{-1} A Q^{-\frac{1}{2}}+\frac{1}{4} I\right]^{\frac{1}{2}} Q^{\frac{1}{2}}+\frac{1}{2} Q \\
& \geq Q^{\frac{1}{2}}\left[Q^{-\frac{1}{2}} A^{T}\left(Q^{-1}+B B^{T}\right)^{-1} A Q^{-\frac{1}{2}}+\frac{1}{4} I\right]^{\frac{1}{2}} Q^{\frac{1}{2}}+\frac{1}{2} Q .
\end{aligned}
$$

This completes the proof.

Furthermore, we propose new lower and upper solution bounds on the basis of Lemma 2.1.

Theorem 2.2 Let $P$ be the positive definite solution of the discrete algebraic Riccati equation (1.3), and $\sigma_{1}^{2}(A)<1+\sigma_{n}^{2}(B) \delta_{1}$, then $P$ has the lower and upper solution bounds

$$
\widetilde{P}_{1} \leq P \leq \widehat{P}_{1}
$$

where

$$
\widetilde{P}_{1} \equiv Q^{\frac{1}{2}}\left[Q^{-\frac{1}{2}} A^{T}\left(Q^{-1}+B B^{T}-A \eta^{-1} A^{T}\right)^{-1} A Q^{-\frac{1}{2}}+\frac{1}{4} I\right]^{\frac{1}{2}} Q^{\frac{1}{2}}+\frac{1}{2} Q
$$

and

$$
\widehat{P}_{1} \equiv Q^{\frac{1}{2}}\left[Q^{-\frac{1}{2}} A^{T}\left(\eta^{-1}+B B^{T}-A Q^{-1} A^{T}\right)^{-1} A Q^{-\frac{1}{2}}+\frac{1}{4} I\right]^{\frac{1}{2}} Q^{\frac{1}{2}}+\frac{1}{2} Q
$$

$\delta_{1}$ and $\eta$ are defined as in Lemma 1.3. 
Proof Applying Lemma 1.3 and (2.18) to (2.17), it is simple to see that

$$
\begin{aligned}
P & =Q^{\frac{1}{2}}\left[Q^{-\frac{1}{2}} A^{T}\left(P^{-1}+B B^{T}-A P^{-1} A^{T}\right)^{-1} A Q^{-\frac{1}{2}}+\frac{1}{4} I\right]^{\frac{1}{2}} Q^{\frac{1}{2}}+\frac{1}{2} Q \\
& \geq Q^{\frac{1}{2}}\left[Q^{-\frac{1}{2}} A^{T}\left(Q^{-1}+B B^{T}-A P^{-1} A^{T}\right)^{-1} A Q^{-\frac{1}{2}}+\frac{1}{4} I\right]^{\frac{1}{2}} Q^{\frac{1}{2}}+\frac{1}{2} Q \\
& \geq Q^{\frac{1}{2}}\left[Q^{-\frac{1}{2}} A^{T}\left(Q^{-1}+B B^{T}-A \eta^{-1} A^{T}\right)^{-1} A Q^{-\frac{1}{2}}+\frac{1}{4} I\right]^{\frac{1}{2}} Q^{\frac{1}{2}}+\frac{1}{2} Q
\end{aligned}
$$

and

$$
\begin{aligned}
P & =Q^{\frac{1}{2}}\left[Q^{-\frac{1}{2}} A^{T}\left(P^{-1}+B B^{T}-A P^{-1} A^{T}\right)^{-1} A Q^{-\frac{1}{2}}+\frac{1}{4} I\right]^{\frac{1}{2}} Q^{\frac{1}{2}}+\frac{1}{2} Q \\
& \leq Q^{\frac{1}{2}}\left[Q^{-\frac{1}{2}} A^{T}\left(\eta^{-1}+B B^{T}-A P^{-1} A^{T}\right)^{-1} A Q^{-\frac{1}{2}}+\frac{1}{4} I\right]^{\frac{1}{2}} Q^{\frac{1}{2}}+\frac{1}{2} Q \\
& \leq Q^{\frac{1}{2}}\left[Q^{-\frac{1}{2}} A^{T}\left(\eta^{-1}+B B^{T}-A Q^{-1} A^{T}\right)^{-1} A Q^{-\frac{1}{2}}+\frac{1}{4} I\right]^{\frac{1}{2}} Q^{\frac{1}{2}}+\frac{1}{2} Q .
\end{aligned}
$$

This completes the proof.

Remark 2.1 For one thing, we point out that $\widetilde{P}_{1} \geq P_{1} \geq Q$. Indeed,

$$
\begin{aligned}
\widetilde{P}_{1} & =Q^{\frac{1}{2}}\left[Q^{-\frac{1}{2}} A^{T}\left(Q^{-1}+B B^{T}-A \eta^{-1} A^{T}\right)^{-1} A Q^{-\frac{1}{2}}+\frac{1}{4} I\right]^{\frac{1}{2}} Q^{\frac{1}{2}}+\frac{1}{2} Q \\
& \geq Q^{\frac{1}{2}}\left[Q^{-\frac{1}{2}} A^{T}\left(Q^{-1}+B B^{T}\right)^{-1} A Q^{-\frac{1}{2}}+\frac{1}{4} I\right]^{\frac{1}{2}} Q^{\frac{1}{2}}+\frac{1}{2} Q=P_{1}
\end{aligned}
$$

and

$$
\begin{aligned}
P_{1} & =Q^{\frac{1}{2}}\left[Q^{-\frac{1}{2}} A^{T}\left(Q^{-1}+B B^{T}\right)^{-1} A Q^{-\frac{1}{2}}+\frac{1}{4} I\right]^{\frac{1}{2}} Q^{\frac{1}{2}}+\frac{1}{2} Q \\
& \geq Q^{\frac{1}{2}}\left(0+\frac{1}{4} I\right)^{\frac{1}{2}} Q^{\frac{1}{2}}+\frac{1}{2} Q=Q
\end{aligned}
$$

which implies that $\widetilde{P}_{1} \geq P_{1} \geq Q$.

Remark 2.2 $P_{1}$ and $\eta$ are two different upper bounds. It is difficult to compare them for the same measure. Further, in Section 5, we offer a numerical example which shows that $P_{1}$ is better than $\eta$ (i.e., $\left.P_{1} \leq \eta\right)$ in a certain case. It is hard to prove that this result holds for the general case in the theory.

\section{On the solution existence uniqueness of the discrete algebraic Riccati equation (1.3)}

In this section, we present a new existence uniqueness condition for the solution of the discrete algebraic Riccati equation (1.3). 
Theorem 3.1 Let $\sigma_{1}^{2}(A)<1+\sigma_{n}^{2}(B) \delta_{1}, A^{T}\left(\widehat{P}_{1}^{-1}+B B^{T}\right)^{-1} A+Q \leq \eta$, and

$$
0<p<1
$$

with

$$
p=\|A\|^{2} \cdot\left\|\widetilde{P}_{1}^{-1}\right\|^{2} \cdot\left(\left\|\widehat{P}_{1}^{-1}+B B^{T}\right\|\right)^{-2},
$$

where $\delta_{1}, \eta$ are defined as in Lemma 1.3, $\widetilde{P}_{1}, \widehat{P}_{1}$ are defined by (2.19). Then the discrete algebraic Riccati equation (1.3) has a unique positive definite solution $P_{0}$, and $\widetilde{P}_{1} \leq P_{0} \leq \widehat{P}_{1}$.

Proof (1) If $\sigma_{1}^{2}(A)<1+\sigma_{n}^{2}(B) \delta_{1}$, using (1.4) and the inequalities

$$
\eta^{-1} \leq Q^{-1}, \quad-A Q^{-1} A^{T} \leq-A \eta^{-1} A^{T},
$$

we get $\widetilde{P}_{1} \leq \widehat{P}_{1}$. Suppose the discrete algebraic Riccati equation (1.3) possesses a positive definite solution, from Theorem 2.2 , then the positive definite solution is in $\left[\widetilde{P}_{1}, \widehat{P}_{1}\right]$, i.e., $\widetilde{P}_{1} \leq P \leq \widehat{P}_{1}$.

(2) Define the map $F(P)=A^{T}\left(P^{-1}+B B^{T}\right)^{-1} A+Q$ and set

$$
P \in \Omega=\left\{P \mid \widetilde{P}_{1} \leq P \leq \widehat{P}_{1}\right\} .
$$

It is obvious that $\Omega$ is a convex, closed, and bounded set and $F(P)$ is continuous on $\Omega$. We consider a Banach space $(\Omega,\|\cdot\|)$, where $\|\cdot\|$ is the spectral norm.

For one thing, since $P \leq \widehat{P}_{1}, A^{T}\left(\widehat{P}_{1}^{-1}+B B^{T}\right)^{-1} A+Q \leq \eta$,

$$
F(P)=A^{T}\left(P^{-1}+B B^{T}\right)^{-1} A+Q \leq A^{T}\left(\widehat{P}_{1}^{-1}+B B^{T}\right)^{-1} A+Q \leq \eta .
$$

For another, since $\widetilde{P}_{1} \leq P$,

$$
F(P)=A^{T}\left(P^{-1}+B B^{T}\right)^{-1} A+Q \geq F\left(\widetilde{P}_{1}\right)=A^{T}\left(\widetilde{P}_{1}^{-1}+B B^{T}\right)^{-1} A+Q \geq Q .
$$

Thus, by Lemma 2.1, we obtain

$$
\begin{aligned}
F(P) & =Q^{\frac{1}{2}}\left[Q^{-\frac{1}{2}} A^{T}\left(P^{-1}+B B^{T}-A(F(P))^{-1} A^{T}\right)^{-1} A Q^{-\frac{1}{2}}+\frac{1}{4} I\right]^{\frac{1}{2}} Q^{\frac{1}{2}}+\frac{1}{2} Q \\
& \geq Q^{\frac{1}{2}}\left[Q^{-\frac{1}{2}} A^{T}\left(Q^{-1}+B B^{T}-A(F(P))^{-1} A^{T}\right)^{-1} A Q^{-\frac{1}{2}}+\frac{1}{4} I\right]^{\frac{1}{2}} Q^{\frac{1}{2}}+\frac{1}{2} Q \\
& \geq Q^{\frac{1}{2}}\left[Q^{-\frac{1}{2}} A^{T}\left(Q^{-1}+B B^{T}-A \eta^{-1} A^{T}\right)^{-1} A Q^{-\frac{1}{2}}+\frac{1}{4} I\right]^{\frac{1}{2}} Q^{\frac{1}{2}}+\frac{1}{2} Q=\widetilde{P}_{1}
\end{aligned}
$$

and

$$
\begin{aligned}
F(P) & =Q^{\frac{1}{2}}\left[Q^{-\frac{1}{2}} A^{T}\left(P^{-1}+B B^{T}-A(F(P))^{-1} A^{T}\right)^{-1} A Q^{-\frac{1}{2}}+\frac{1}{4} I\right]^{\frac{1}{2}} Q^{\frac{1}{2}}+\frac{1}{2} Q \\
& \leq Q^{\frac{1}{2}}\left[Q^{-\frac{1}{2}} A^{T}\left(\eta^{-1}+B B^{T}-A(F(P))^{-1} A^{T}\right)^{-1} A Q^{-\frac{1}{2}}+\frac{1}{4} I\right]^{\frac{1}{2}} Q^{\frac{1}{2}}+\frac{1}{2} Q \\
& \leq Q^{\frac{1}{2}}\left[Q^{-\frac{1}{2}} A^{T}\left(\eta^{-1}+B B^{T}-A Q^{-1} A^{T}\right)^{-1} A Q^{-\frac{1}{2}}+\frac{1}{4} I\right]^{\frac{1}{2}} Q^{\frac{1}{2}}+\frac{1}{2} Q=\widehat{P}_{1} .
\end{aligned}
$$


Consequently, for $P \in \Omega$, we get $\widetilde{P}_{1} \leq F(P) \leq \widehat{P}_{1}$.

Thus $F(\Omega) \subseteq \Omega$.

For arbitrary $P_{1}, P_{2} \in \Omega$,

$$
\begin{aligned}
& F\left(P_{1}\right)=A^{T}\left(P_{1}^{-1}+B B^{T}\right)^{-1} A+Q, \\
& F\left(P_{2}\right)=A^{T}\left(P_{2}^{-1}+B B^{T}\right)^{-1} A+Q .
\end{aligned}
$$

Consequently,

$$
\begin{aligned}
F\left(P_{1}\right)-F\left(P_{2}\right) & =A^{T}\left[\left(P_{1}^{-1}+B B^{T}\right)^{-1}-\left(P_{2}^{-1}+B B^{T}\right)^{-1}\right] A \\
& =A^{T}\left\{\left(P_{1}^{-1}+B B^{T}\right)^{-1}\left[\left(P_{2}^{-1}+B B^{T}\right)-\left(P_{1}^{-1}+B B^{T}\right)\right]\left(P_{2}^{-1}+B B^{T}\right)^{-1}\right\} A \\
& =A^{T}\left(P_{1}^{-1}+B B^{T}\right)^{-1}\left(P_{2}^{-1}-P_{1}^{-1}\right)\left(P_{2}^{-1}+B B^{T}\right)^{-1} A \\
& =A^{T}\left(P_{1}^{-1}+B B^{T}\right)^{-1} P_{1}^{-1}\left(P_{1}-P_{2}\right) P_{2}^{-1}\left(P_{2}^{-1}+B B^{T}\right)^{-1} A .
\end{aligned}
$$

Since $\widetilde{P}_{1} \leq P \leq \widehat{P}_{1}$, according to Lemma 1.6, we have

$$
\begin{aligned}
\left\|F\left(P_{1}\right)-F\left(P_{2}\right)\right\|= & \left\|A^{T}\left(P_{1}^{-1}+B B^{T}\right)^{-1} P_{1}^{-1}\left(P_{1}-P_{2}\right) P_{2}^{-1}\left(P_{2}^{-1}+B B^{T}\right)^{-1} A\right\| \\
\leq & \left\|A^{T}\right\| \cdot\|A\| \cdot\left\|P_{1}^{-1}\right\| \cdot\left\|P_{2}^{-1}\right\| \cdot\left\|\left(P_{1}^{-1}+B B^{T}\right)^{-1}\right\| \\
& \cdot\left\|\left(P_{2}^{-1}+B B^{T}\right)^{-1}\right\| \cdot\left\|P_{1}-P_{2}\right\| \\
\leq & \left\|A^{T}\right\| \cdot\|A\| \cdot\left\|\widetilde{P}_{1}^{-1}\right\| \cdot\left\|\widetilde{P}_{1}^{-1}\right\| \cdot\left(\left\|\widehat{P}_{1}^{-1}+B B^{T}\right\|\right)^{-1} \\
& \cdot\left(\left\|\widehat{P}_{1}^{-1}+B B^{T}\right\|\right)^{-1} \cdot\left\|P_{1}-P_{2}\right\| \\
= & \|A\|^{2} \cdot\left\|\widetilde{P}_{1}^{-1}\right\|^{2} \cdot\left(\left\|\widehat{P}_{1}^{-1}+B B^{T}\right\|\right)^{-2} \cdot\left\|P_{1}-P_{2}\right\| \\
= & p\left\|P_{1}-P_{2}\right\| .
\end{aligned}
$$

As $p<1$, thus the map $F(P)$ is a contraction map in $\Omega$. In the light of Lemma 1.5, the map $F(P)$ has a unique fixed point in $\Omega$.

Combing (1) and (2) shows that (1.3) has a unique positive definite solution $P_{0}$, and $\widetilde{P}_{1} \leq$ $P_{0} \leq \widehat{P}_{1}$.

\section{A fixed point iteration algorithm for the solution of the discrete algebraic \\ Riccati equation (1.3)}

In this section, based on Theorem 3.1, we present a fixed point iteration algorithm for the solution of the discrete algebraic Riccati equation (1.3).

For arbitrary given $P^{(0)} \in \Omega=\left\{P \mid \widetilde{P}_{1} \leq P \leq \widehat{P}_{1}\right\}$, we construct the matrix sequence

$$
P^{(k+1)}=A^{T}\left[\left(P^{(k)}\right)^{-1}+B B^{T}\right]^{-1} A+Q, \quad k=0,1, \ldots
$$

Next, we prove $P^{(k)}$ is convergent and converges to the exact solution of the discrete algebraic Riccati equation (1.3).

Theorem 4.1 If the conditions of Theorem 3.1 are satisfied, then the sequence $P^{(k)}$ given by (4.1) is convergent and converges to the unique positive definite solution $P_{0}$ of the discrete algebraic Riccati equation (1.3). 
Proof For $P^{(0)} \in \Omega$, in terms of (4.1), for $k \in N^{+}$, we obtain a sequence $\left\{P^{(k)}\right\}$, then

$$
\begin{aligned}
P^{(k)}-P^{(k-1)}= & A^{T}\left\{\left[\left(P^{(k-1)}\right)^{-1}+B B^{T}\right]^{-1}-\left[\left(P^{(k-2)}\right)^{-1}+B B^{T}\right]^{-1}\right\} A \\
= & A^{T}\left\{\left[\left(P^{(k-1)}\right)^{-1}+B B^{T}\right]^{-1}\left\{\left[\left(P^{(k-2)}\right)^{-1}+B B^{T}\right]-\left[\left(P^{(k-1)}\right)^{-1}+B B^{T}\right]\right\}\right. \\
& \left.\times\left[\left(P^{(k-2)}\right)^{-1}+B B^{T}\right]^{-1}\right\} A \\
= & A^{T}\left[\left(P^{(k-1)}\right)^{-1}+B B^{T}\right]^{-1}\left[\left(P^{(k-2)}\right)^{-1}-\left(P^{(k-1)}\right)^{-1}\right]\left[\left(P^{(k-2)}\right)^{-1}+B B^{T}\right]^{-1} A \\
= & A^{T}\left[\left(P^{(k-1)}\right)^{-1}+B B^{T}\right]^{-1}\left(P^{(k-1)}\right)^{-1}\left(P^{(k-1)}-P^{(k-2)}\right)\left(P^{(k-2)}\right)^{-1} \\
& \times\left[\left(P^{(k-2)}\right)^{-1}+B B^{T}\right]^{-1} A .
\end{aligned}
$$

In a similar way to the proof of (3.2), we obtain

$$
\left\|P^{(k)}-P^{(k-1)}\right\| \leq p\left\|P^{(k-1)}-P^{(k-2)}\right\| .
$$

Applying mathematical induction, it is easy to get

$$
\left\|P^{(k)}-P^{(k-1)}\right\| \leq p^{k-1}\left\|P^{(1)}-P^{(0)}\right\| .
$$

Because $0<p<1$, thus $\lim _{k \rightarrow \infty} p^{k}=0$. Then, $\forall \varepsilon>0, \exists N_{1} \in N^{+}, \forall k>N_{1}$, we get

$$
p^{k}<\frac{\varepsilon(1-p)}{\left\|P^{(1)}-P^{(0)}\right\|}
$$

Consequently, $\forall k>N_{1}, \forall v \in N^{+}$, using the triangular inequality, (4.2), and (4.3), we obtain

$$
\begin{aligned}
\left\|P^{(k+v)}-P^{(k)}\right\| & \leq\left\|P^{(k+v)}-P^{(k+v-1)}\right\|+\left\|P^{(k+v-1)}-P^{(k+v-2)}\right\|+\cdots+\left\|P^{(k+1)}-P^{(k)}\right\| \\
& \leq\left(p^{k+v-1}+p^{k+v-2}+\cdots+p^{k}\right)\left\|P^{(1)}-P^{(0)}\right\| \\
& =\frac{p^{k}-p^{k+v}}{1-p}\left\|P^{(1)}-P^{(0)}\right\| \\
& <\frac{p^{k}}{1-p}\left\|P^{(1)}-P^{(0)}\right\| \\
& <\varepsilon .
\end{aligned}
$$

Hence $P^{(k)}$ is a Cauchy sequence in $\Omega$, then $P^{(k)}$ is convergent. Denote $\lim _{k \rightarrow \infty} P^{(k)}=P_{0}$. Let $k \rightarrow \infty$ in (4.1), then

$$
P_{0}=A^{T}\left(P_{0}^{-1}+B B^{T}\right)^{-1} A+Q
$$

This shows that $P_{0}$ is a solution of (1.3). According to Theorem 3.1, $P_{0}$ is the unique positive definite solution of the discrete algebraic Riccati equation (1.3). This completes the proof.

By Theorem 4.1, define $P^{(k)}$ as the $k$ th iterative solution of the discrete algebraic Riccati equation (1.3). For the iterative solution $P^{(k+1)}$ and $P^{(k)}$, by (4.2), for any small $\varepsilon>0$, we 
have the error estimation

$$
\left\|P^{(k+1)}-P^{(k)}\right\| \leq p^{k}\left\|P^{(1)}-P^{(0)}\right\| \leq p^{k}\left\|\widehat{P}_{1}-\widetilde{P}_{1}\right\|<\varepsilon .
$$

In the case of the precision $\varepsilon$ is permitted, we have the following algorithm.

\section{Algorithm 4.1}

Input: For the discrete algebraic Riccati equation (1.3), given $A, B, Q$.

Output: $P^{(k)}$.

Step 1: Compute $\widetilde{P}_{1}$ and $\widehat{P}_{1}$.

Step 2: Compute $p$, if $0<p<1$, go to Step 3; otherwise, stop.

Step 3: Set $P^{(0)} \in \Omega=\left\{P \mid \widetilde{P}_{1} \leq P \leq \widehat{P}_{1}\right\}$.

Step 4: Compute

$$
P^{(k+1)}=A^{T}\left[\left(P^{(k)}\right)^{-1}+B B^{T}\right]^{-1} A+Q, \quad k=0,1, \ldots
$$

Step 5: For any small $\varepsilon>0$ and $k \in N^{+}$, if $\left\|P^{(k+1)}-P^{(k)}\right\|<\varepsilon$, stop; otherwise, let $k=k+1$, go to Step 4 .

\section{A numerical example}

In this section, we demonstrate the effectiveness of our results by the following real application example. In many engineering fields such as solid mechanics, quantum mechanics, parameter identification and automatic control, we often need to study the system stability and optimal control of linear discrete systems. Sometimes, these problems reduce to discussing the symmetric positive definite solution of the corresponding discrete algebraic Riccati matrix equations (1.2) and (1.3). The whole process of the example is carried out on Matlab 7.1 and the precision is $10^{-8}$.

Example 5.1 Consider the following linear discrete system (see [2]):

$$
x(t+1)=A x(t)+B u(t),
$$

where $x(t) \in \mathbb{R}^{n}$ is the state variable, $u(t) \in \mathbb{R}^{m}$ is the input variable, $A \in \mathbb{R}^{n \times n}$ is the system matrix, $B \in \mathbb{R}^{n \times m}$ is the input matrix. Here, we choose

$$
\begin{aligned}
A & =\left(\begin{array}{ccc}
0.02 & 0.05 & 0.1 \\
0.03 & 0.04 & 0 \\
0.06 & -0.1 & 0.05
\end{array}\right), \\
B & =\left(\begin{array}{ccc}
0.1 & 0 & 0.2 \\
0.3 & 0.4 & 0 \\
0 & 0.15 & 0.16
\end{array}\right) .
\end{aligned}
$$

The quadratic performance index of (5.1) is

$$
J=\frac{1}{2} \sum_{t=0}^{\infty}\left[x^{T}(t) Q x(t)+u^{T}(t) u(t)\right],
$$


where

$$
Q=\left(\begin{array}{ccc}
0.6 & 0.12 & 0.1 \\
0.12 & 0.4 & 0.05 \\
0.1 & 0.05 & 0.3
\end{array}\right)
$$

is symmetric positive definite.

In order to seek the optimal control such that the quadratic performance index (5.2) attains the minimum, we use the minimum principle. Choose the Hamiltonian function as

$$
H(t)=\frac{1}{2} x^{T}(t) Q x(t)+\frac{1}{2} u^{T}(t) u(t)+\chi^{T}(t+1)[A x(t)+B u(t)] .
$$

Then we can obtain the canonical equation

$$
\chi(t)=\frac{\partial H(t)}{\partial x(t)}=Q x(t)+A^{T} \chi(t+1)
$$

and the control equation

$$
\frac{\partial H(t)}{\partial u(t)}=u(t)+B^{T} \chi(t+1)=0
$$

i.e.,

$$
u(t)=-B^{T} \chi(t+1) .
$$

Suppose

$$
\chi(t)=P x(t) .
$$

Substituting (5.5) into the canonical equation (5.3) yields

$$
P x(t)=Q x(t)+A^{T} P x(t+1) .
$$

Using the state equation (5.1) and the control equation (5.4), we have

$$
x(t+1)=A x(t)+B u(t)=A x(t)-B B^{T} P x(t+1),
$$

then

$$
x(t+1)=\left(I+B B^{T} P\right)^{-1} A x(t) .
$$

Substituting (5.7) into (5.6), eliminating $x(t)$ on both sides of the equality, applying the Sherman-Morrison-Woodbury equality

$$
(I+S T)^{-1}=I-S(I+T S)^{-1} T,
$$


we get the discrete algebraic Riccati equation (1.3),

$$
P=A^{T} P A-A^{T} P B\left(I+B^{T} P B\right)^{-1} B^{T} P A+Q .
$$

In terms of (5.4) and (5.5), by (5.8), we obtain the optimal control

$$
u(t)=-\left(I+B^{T} P B\right)^{-1} B^{T} P A x(t)
$$

such that the quadratic performance index (5.2) attains a minimum, where $P$ is the symmetric positive definite solution of the discrete algebraic Riccati equation (1.3).

Obviously, $\sigma_{1}^{2}(A)<1+\sigma_{n}^{2}(B) \delta_{1}$, then the upper matrix bound for the solution $P$ of the discrete algebraic Riccati equation (1.3) by (1.4) is

$$
\eta=\left(\begin{array}{ccc}
0.60339617 & 0.11736623 & 0.10346548 \\
0.11736623 & 0.40977266 & 0.05000000 \\
0.10346548 & 0.05000000 & 0.30866371
\end{array}\right),
$$

leading to

$$
\lambda_{1}(\eta)=0.69307554, \quad \lambda_{2}(\eta)=0.35445560, \quad \lambda_{3}(\eta)=0.27430140 .
$$

The lower matrix bound for the solution $P$ of the discrete algebraic Riccati equation (1.3) found by (2.13) is

$$
P_{1}=\left(\begin{array}{ccc}
0.60205607 & 0.11950250 & 0.10293022 \\
0.11950250 & 0.40416982 & 0.05112541 \\
0.10293022 & 0.05112541 & 0.30720800
\end{array}\right) \text {, }
$$

leading to

$$
\lambda_{1}\left(P_{1}\right)=0.69252521, \quad \lambda_{2}\left(P_{1}\right)=0.34803838, \quad \lambda_{3}\left(P_{1}\right)=0.27287030 .
$$

The lower and upper matrix bounds for the solution $P$ of the discrete algebraic Riccati equation (1.3) found by (2.19) are

$$
\widetilde{P}_{1}=\left(\begin{array}{ccc}
0.60204980 & 0.11953871 & 0.10295826 \\
0.11953871 & 0.40415536 & 0.05121050 \\
0.10295826 & 0.05121050 & 0.30729715
\end{array}\right) \text {, }
$$

leading to

$$
\begin{aligned}
& \lambda_{1}\left(\widetilde{P}_{1}\right)=0.69258489, \quad \lambda_{2}\left(\widetilde{P}_{1}\right)=0.34800103, \quad \lambda_{3}\left(\widetilde{P}_{1}\right)=0.27291639, \\
& \widehat{P}_{1}=\left(\begin{array}{lll}
0.60209132 & 0.11949876 & 0.10300401 \\
0.11949876 & 0.40422207 & 0.05115031 \\
0.10300401 & 0.05115030 & 0.30738615
\end{array}\right),
\end{aligned}
$$


leading to

$$
\begin{aligned}
& \lambda_{1}\left(\widehat{P}_{1}\right)=0.69261472, \quad \lambda_{2}\left(\widehat{P}_{1}\right)=0.34808987, \\
& \lambda_{3}\left(\widehat{P}_{1}\right)=0.27299495
\end{aligned}
$$

Obviously,

$$
\widetilde{P}_{1} \geq P_{1}, \quad \eta \geq \widehat{P}_{1}
$$

This shows that the lower bound $\widetilde{P}_{1}$ is better than $P_{1}$, and the upper bound $\widehat{P}_{1}$ is better than $\eta$.

By computation, obviously, $\widetilde{P}_{1} \leq \widehat{P}_{1}, A^{T}\left(\widehat{P}_{1}^{-1}+B B^{T}\right)^{-1} A+Q \leq \eta$, and

$$
0<p=0.01643822<1 .
$$

According to Theorem 3.1, the discrete algebraic Riccati equation (1.3) has a unique positive definite solution $P_{0}$, and $\widetilde{P}_{1} \leq P_{0} \leq \widehat{P}_{1}$.

Let $e(k)=\left\|P^{(k+1)}-P^{(k)}\right\|$ be the iteration error at the $k$ th iteration, $k$ denote the iteration number, where we choose $\varepsilon=10^{-8}$ and $P^{(0)}=\widetilde{P}_{1}$ and $P^{(0)}=Q$, respectively. Then Algorithm 4.1 and Theorem 3.1 of [3] need 4 and 5 iteration steps, respectively, to converge to the iteration solution of the discrete algebraic Riccati equation (1.3) as follows:

$$
P=\left(\begin{array}{ccc}
0.60208663 & 0.11950317 & 0.10300002 \\
0.11950317 & 0.40420121 & 0.05116389 \\
0.10300002 & 0.05116389 & 0.30735947
\end{array}\right)
$$

The relation between iteration step and iteration error is shown in Table 1 and Figure 1. Obviously, Table 1 and Figure 1 show that compared to Theorem 3.1 of [3], Algorithm 4.1 has less errors and less iteration steps.

Thus, by (5.9), we can have the optimal control

$$
\begin{aligned}
u(t) & =-\left(I+B^{T} P B\right)^{-1} B^{T} P A x(t) \\
& =\left(\begin{array}{lll}
0.00644824 & 0.00704508 & 0.00957459 \\
0.00909563 & 0.00265056 & 0.00810075 \\
0.00724564 & 0.00089025 & 0.01614435
\end{array}\right) x(t)
\end{aligned}
$$

such that the quadratic performance index (5.2) attains a minimum.

Table 1 Iteration errors $\left(\varepsilon=10^{-8}\right)$

\begin{tabular}{lllll}
\hline \multicolumn{2}{c}{$\boldsymbol{P}_{\mathbf{0}}=\widehat{\boldsymbol{P}}_{\mathbf{1}}$} & & \multicolumn{2}{l}{$\boldsymbol{P}_{\mathbf{0}}=\mathbf{Q}$} \\
\cline { 1 - 2 } $\boldsymbol{k}$ & $\boldsymbol{e}(\boldsymbol{k})$ & & $\boldsymbol{e}(\boldsymbol{k})$ \\
\hline 1 & 0.00198010 & & 0.00881228 \\
2 & $6.53368839 \times 10^{-6}$ & & $1.29922911 \times 10^{-4}$ \\
3 & $5.18447904 \times 10^{-8}$ & 3 & $1.54679034 \times 10^{-6}$ \\
4 & $6.25516090 \times 10^{-10}$ & 4 & $1.94930367 \times 10^{-8}$ \\
& & 5 & $1.90929712 \times 10^{-10}$ \\
\hline
\end{tabular}


Figure 1 Iteration errors. The red line and blue line denote $P^{(0)}=\widetilde{P}_{1}=\psi$ and $P^{(0)}=Q$, respectively.

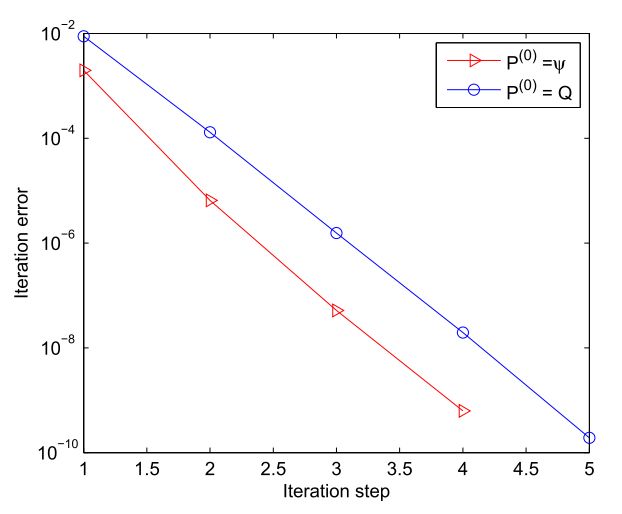

Competing interests

The authors declare that they have no competing interests.

Authors' contributions

All authors contributed equally to the writing of this paper. All authors read and approved the final manuscript.

\section{Acknowledgements}

The work was supported in part by National Natural Science Foundation of China (11571292, 11471279), National Natural Science Foundation for Youths of China (11401505), the Key Project of National Natural Science Foundation of China (91430213), the General Project of Hunan Provincial Natural Science Foundation of China (2015JJ2134), and the General Project of Hunan Provincial Education Department of China (15C1320).

\section{Received: 14 May 2015 Accepted: 28 September 2015 Published online: 09 October 2015}

\section{References}

1. Bernstein, DS: Matrix Mathematics: Theory, Facts and Formulas with Application to Linear Systems Theory. Princeton University Press, Princeton (2005)

2. Kojima, C, Takaba, K, Kaneko, O, Rapisarda, P: A characterization of solutions of the discrete-time algebraic Riccati equation based on quadratic difference forms. Linear Algebra Appl. 416, 1060-1082 (2006)

3. Komaroff, N: Iterative matrix bounds and computational solutions to the discrete algebraic Riccati equation. IEEE Trans. Autom. Control 39, 1676-1678 (1994)

4. Lee, $\mathrm{CH}, \mathrm{Hsien}, \mathrm{TL}$ : New solution bounds for the discrete algebraic matrix Riccati equation. Optim. Control Appl. Methods 20,113-125 (1999)

5. Garloff, J: Bounds for the eigenvalues of the solution of the discrete Riccati and Lyapunov equations and the continuous Lyapunov equation. Int. J. Control 43, 423-431 (1986)

6. Lee, $\mathrm{CH}$ : A unified approach to eigenvalue bounds of the solution of the Riccati equation. J. Chin. Inst. Eng. 27, 431-437 (2004)

7. Fang, Y, Loparo, KA: Stabilization of continuous-time jump linear systems. IEEE Trans. Autom. Control 47, 1590-1603 (2002)

8. Dragan, $\mathrm{V}$ : The linear quadratic optimization problem for a class of singularly stochastic systems. Int. J. Innov. Comput. Inf. Control 1, 53-63 (2005)

9. Assimakis, N, Roulis, S, Lainiotis, D: Recursive solutions of the discrete time Riccati equation. Neural Parallel Sci. Comput. 11, 343-350 (2003)

10. Saberi, SA: The discrete algebraic Riccati equation and linear matrix inequality. Linear Algebra Appl. 274, 317-365 (1998)

11. Assimakis, N, Adam, M: Kalman filter Riccati equation for the prediction, estimation and smoothing error covariance matrices. ISRN Comput. Math. 13, Article ID 249594 (2013). doi:10.1155/2013/249594

12. Assimakis, N, Adam, M: Iterative and algebraic algorithms for the computation of the steady state Kalman filter gain ISRN Appl. Math. 2014, Article ID 417623 (2014). doi:10.1155/2014/417623

13. Chiang, C-Y, Fan, H-Y, Lin, W-W: A structured doubling algorithm for discrete-time algebraic Riccati equations with singular control weighting matrices. Taiwan. J. Math. 14(3A), 933-954 (2010)

14. Choi, $\mathrm{HH}$ : Upper matrix bounds for the discrete algebraic Riccati matrix equation. IEEE Trans. Autom. Control 46 , 504-508 (2001)

15. Kim, SW, Park, PG: Matrix bounds of the discrete ARE solution. Syst. Control Lett. 36, 15-20 (1999)

16. Gao, L, Xue, A, Sun, Y: Comments on 'Upper matrix bounds for the discrete algebraic Riccati matrix equation'. IEEE Trans. Autom. Control 47, 1212-1213 (2002)

17. Komaroff, N: Upper bounds for the solution of the discrete Riccati equation. IEEE Trans. Autom. Control 37, 1370-1373 (1992)

18. Lee, $\mathrm{CH}$, Chang, YC: Solution bounds for the discrete Riccati equation and its applications. J. Optim. Theory Appl. 99 443-463 (1998)

19. Lee, $\mathrm{CH}$ : On the matrix bounds for the solution matrix of the discrete algebraic Riccati equation. IEEE Trans. Circuits Syst. I 43, 402-407 (1996) 
20. Lee, $\mathrm{CH}$ : Upper matrix bound of the solution for the discrete Riccati equation. IEEE Trans. Autom. Control 42, 840-842 (1997)

21. Lee, CH: Matrix bounds of the solutions of the continuous and discrete Riccati equations - a unified approach. Int J. Control 76, 635-642 (2003)

22. Lee, $\mathrm{CH}$ : Upper and lower bounds of the solutions of the discrete algebraic Riccati and Lyapunov matrix equations. Int. J. Control 68, 579-598 (1997)

23. Liu, J-Z, Zhang, J: The existence uniqueness and the fixed iterative algorithm of the solution for the discrete coupled algebraic Riccati equation. Int. J. Control 84(8), 1430-1441 (2011)

24. Liu, J-Z, Zhang, J: Upper solution bounds of the continuous coupled algebraic Riccati matrix equation. Int. J. Control 84(4), 726-736 (2011)

25. Liu, J-Z, Zhang, J: New upper matrix bounds of the solution for perturbed continuous coupled algebraic Riccati matrix equation. Int. J. Control. Autom. Syst. 10(6), 1254-1259 (2012)

26. Zhang, J, Liu, JZ: New matrix bounds, an existence uniqueness and a fixed-point iterative algorithm for the solution of the unified coupled algebraic Riccati equation. Int. J. Comput. Math. 89(4), 527-542 (2012)

27. Zhang, J, Liu, JZ: Lower solution bounds of the continuous coupled algebraic Riccati matrix equation. Int. J. Control. Autom. Syst. 10(6), 1273-1278 (2012)

28. Kim, SW, Park, PG, Kwon, WH: Lower bounds for the trace of the solution of the discrete algebraic Riccati equation. IEEE Trans. Autom. Control 38, 12-14 (1993)

29. Komaroff, N, Shahian, B: Lower summation bounds for the discrete Riccati and Lyapunov equations. IEEE Trans. Autom. Control 37, 1078-1080 (1992)

30. Patel, RV, Toda, M: On norm bounds for algebraic Riccati and Lyapunov equations. IEEE Trans. Autom. Control 23, $87-88(1978)$

31. Hasanov, VI, Ivanov, IG, Uhlig, F: Improved perturbation estimates for the matrix equations $X \pm A^{*} X^{-1} A=Q$. Linear Algebra Appl. 379, 113-135 (2004)

32. Hasanov, VI: Notes on two perturbation estimates of the extreme solutions to the equations $X \pm A^{*} X^{-1} A=Q$. Appl. Math. Comput. 216, 1355-1362 (2010)

33. Zhang, F-Z: The Schur Complement and Its Applications. Springer, New York (2005)

34. Rudin, W: Functional Analysis. McGraw-Hill, New York (1973)

35. Berinde, $\mathrm{V}$ : A common fixed point theorem for compatible quasi contractive self mappings in metric spaces. Appl. Math. Comput. 213, 348-354 (2009)

36. Zhao, W-L, Li, H-K, Liu, X-T, Xu, F-Y: Necessary and sufficient conditions for the existence of a Hermitian positive definite solution of a type of nonlinear matrix equations. Math. Probl. Eng. 2009, Article ID 672695 (2009)

\section{Submit your manuscript to a SpringerOpen ${ }^{\circ}$ journal and benefit from:}

- Convenient online submission

- Rigorous peer review

- Immediate publication on acceptance

- Open access: articles freely available online

- High visibility within the field

- Retaining the copyright to your article 